Ann. Sci. forest., 1982, 39 (3), 289-306

\title{
Economie de l'eau d'un écosystème à Pinus pinea L. du littoral méditerranéen
}

\author{
M. IBRAHIM *, M. RAPP **, P. LOSSAINT ** \\ * Nouvelle cidresse : University of Tanta, Fac. of Agriculture, Dpt of Soil Science, \\ Kajr El Cheikh, Egypte \\ ** C.N.R.S., C.E.P.E. L. Emberger, B.P. 5051, F 34033 Montpellier Cedex
}

\begin{abstract}
Résumé
Dans le cadre de recherches intégrées sur l'écologie de Pinus pinea, on a étudié, cr.tre autre, la dynamique de l'eau dans un peuplement de cette espèce.

Les principaux paramètres suivants ont été déterminés : apport, interception, eau du sol, drainage, fluctuation de la nappe phréatique, recharge du sol par la nappe et enfin tr.ınspiration par la méthode de l'eau tritiée.

A l'aide des données obtenues, on a abouti à l'établissement du bilan hydrique du système pour deux saisons de végétation.

On note que les valeurs de l'ćvapotranspiration réelle sont proches de l'évaporation potentielle, ce qui explique les facilités d'implantation et de croissance de Pinus pinea dans lcs conditions où cette essence bénéficie toujours d'une alimentaiton hydrique optimale grâce à la présence d'une nappe aquifère facilsment accessible.
\end{abstract}

Mots-clés

Ecosystème, bilan hydrique, Pinus pinea $L$.

\section{1. - Introduction}

La connaissance de la dynamique et du bilan de l'eau dans les biocénoses terrestres est d'un intérêt primordial. En effet, la disponibilité et l'économie de l'eau conditionnent non seulement toute possibilité de colonisation du sol, mais encore, les phénomènes de croissance et de productivité du tapis végétal.

Au niveau du fonctionnement de l'écosystème, l'eau est un vecteur important pour les échanges et les transferts des substances et un facteur essentiel de l'activité biologique et microbiologique du système. 
Si les études globales du cycle de l'eau sont relativement nombreuses dans le cas des agroécosystèmes, l'on constate qu'elles sont rares en milieu forestier et pratiquement inexistantes en région méditerranéenne. Parmi les rares études complètes entreprises en forêt l'on peut citer les travaux de RuTTER $(1964,1966)$ en GrandeBretagne, ou de Blum, Munnich \& Zimmermann (1966) en Allemagne. Un certain nombre de recherches ont également été réalisées dans le cadre du P.B.I., entre autres celles de Schnock (1970) en Belgique, Benecke \& MAYer (1971) en Allemagne, Molchanov (1971) en U.R.S.S., Huttel (1972) en Côte-d'Ivoire, EtTehad et al. (1973) en région méditerranéenne. Plus récemment encore, l'on trouve les travaux de Halldin \& Jansson (1976) en Suède et Fardjah \& Lemee (1980) en France.

Le présent travail porte sur le bilan hydrique d'un peuplement de Pinus pinea $\mathbf{L}$. Il constitue la synthèse d'un ensemble d'investigations entreprises sur le cycle et la dynamique de l'eau de ce peuplement (IBRAHIM, 1979), qui fait l'objet par ailleurs d'une étude détaillée de son fonctionnement au niveau des cycles du carbone, de l'azote et des éléments minéraux (RAPP, Leclerc, Lossaint, 1979).

\section{2. - L'écosystème étudié}

Le peuplement qui couvre une superficie d'une trentaine d'hectares est situé en Petite Camargue, au lieu-dit Petit-Saint-Jean, à l'ouest du delta du Rhône. Il est constitué presque uniquement de Pinus pinea L. âgés de 30 à 35 ans, de 11 à $12 \mathrm{~m}$ de hauteur, et ayant une densité de 800 pieds à l'hectare.

Il provient d'une régénération spontanée après une coupe à blanc.

Le sol s'est formé sur d'anciennes dunes, stabilisées par les pins. Il a été décrit en détail par IBRAHIM \& RAPP (1979) et se compose d'une litière plus ou moins épaisse (3 à $12 \mathrm{~cm}$ ) reposant sur un horizon $A_{1}$ d'épaisseur variable $(0-20 \mathrm{~cm})$, recouvrant lui-même un horizon $C$ sableux. Ce dernier est entrecoupé dans certaines zones, entre -20 et $-60 \mathrm{~cm}$, par un niveau relativement plus riche en substances organominérales, représentant certainement le sol d'un ancien marais recouvert par le sable dunaire. D'après la classification française des sols, c'est un régosol peu évolué, sur sable d'origine éolienne.

\section{3. - Le cycle de l'eau au niveau de la station étudiée}

Le cycle de l'eau d'un écosystème s'articule généralement autour d'une série d'apports, de pertes et de fluctuations internes au système. Dans le cas d'un écosystème forestier, la couverture végétale a une influence considérable. Elle intercepte et ráévapore rapidement vers l'atmosphère une partie de l'eau de pluie. L'eau non interceptée s'infiltre dans le sol, ou ruisselle à sa surface. Une partie de l'eau d'infiltration est retenue dans le sol, et peut y remonter jusqu'à la surface par capillarité, et être ainsi évaporée (évaporation physique). Une autre partie est prélevée par les racines des plantes. Celle-ci, amenée aux feuilles, est transpirée dans l'atmosphère. Enfin, lorsque la fraction de l'eau de pluie infiltrée dépasse la capacité maximale en eau 
du sol, elle percole jusqu'à la nappe aquifère (drainage). Par contre, quand la nappe aquifère est élevée, l'eau peut entrer dans l'écosystème par remontée capillaire à partir des horizons sous-jacents (recharge par la nappe). Ceci est le cas de la station étudiée, dans laquelle un dernier paramètre vient se greffer sur le schéma général : la présence d'un canal artificiel alimentant ou drainant la nappe selon les besoins en eau de l'agriculture environnante.

Le bilan hydrique final peut s'exprimer par la relation suivante :

où :

$$
\mathrm{Pi}+\mathrm{N}=\mathrm{ETR}+\mathrm{I}+\mathrm{D}+\Delta \mathrm{S}
$$

Pi : précipitations incidentes,

$\mathrm{N}$ : apports par la nappe,

ETR : évapotranspiration,

I : interception (eau mouillant la surface des arbres et réévaporéc à la fin de l'ondés),

D : drainage,

$\Delta S \quad$ : variation du stock d'eau dans le sol.

Dans ce bilan nous négligeons l'eau fixée sur le matériel biologique, donc l'hydromasse de la phytocénose, car elle ne varie que très peu avec le temps (ScHNock, 1972). De même, le ruissellement peut aussi être considéré comme inexistant dans cette station, horizontale dans son ensemble, malgré quelques dunes et présentant par ailleurs une très grande porosité du sol.

\section{4. - Méthodes d'études}

\subsection{Précipitations et interception}

Les précipitations incidentes $(\mathrm{Pi})$ ont été mesurées par trois pluviomètres de $660 \mathrm{~cm}^{2}$ d'ouverture et un pluviographe enregistreur placés en dehors du bois.

L'égouttement à travers le feuillage $(\mathrm{Eg})$ a également été mesuré à l'aide de pluviomètres, identiques à ceux utilisés à découvert, mais au nombre de 22 , disposés entre les arbres. Ce dispositif a été complété par quatre pluviomètres auge, de $2,5 \mathrm{~m}$ de long et de $2500 \mathrm{~cm}^{2}$ de surface de captage. Ce dernier dispositif permet une meilleure intégration des fluctuations spatiales résultant de I'hétérogénéité du couvert (RAPP \& IBRAHIM, 1978).

Quant à l'écoulement $(E c)$, il a été mesuré sur 8 arbres, qui par leur surface terrière, leur diamètre moyen et leur répartition spatiale se rapprochent le plus des caractéristiques du peuplement. Cette approche permet de transformer directement les volumes d'eau recueillis en hauteur de précipitations.

L'interception du peuplement est calculé d'après la relation :

$$
\mathrm{I}=\mathrm{Pi}-(\mathrm{Eg}+\mathrm{Ec})
$$


Au bout de trois années continues d'observation, comportant 71 semaines à un ou plusieurs événements pluvieux, l'on a établi l'équation de régression suivante :

$$
\mathrm{I}=0,232 \mathrm{Pi}+1,369(\mathrm{r}=0,910)
$$

qui donne directement la pluie interceptée par rapport à la pluie incidente.

\subsection{Teneur en eau du sol}

Elle a été déterminée par la méthode neutronique (Couchat, 1974), en utilisant un humidimètre à neutrons.

Une série de 13 tubes d'accès en duralumin a été implantée jusqu'à $1,5 \mathrm{~m}$ de profondeur, niveau qui correspond à la hauteur inférieure de la surface de la nappe en été. Huit ont été alignés, tous les 10 mètres, à travers la placette d'expérimentation, 5 ont été placés de manière à étudier l'influence des arbres sur le taux d'humidité du sol.

L'application de l'analyse factorielle en composantes principales, aux observations effectuées hebdomadairement durant 15 mois sur l'ensemble des 13 points de mesure a permis de sélectionner les tubes les plus représentatifs de la variation du stock d'eau moyen de l'ensemble de la parcelle étudiée.

\subsection{Drainage et remontée capillaire}

Les flux profonds sont calculés à partir de deux types d'obscrvations effectuées simultanément dans une zone de sol, dépourvue de racines de pins. A cet effet, une aire de $6 \mathrm{~m}^{2}$ a été isolée, sous forêt, jusqu'à $1,5 \mathrm{~m}$ de profondeur, par des plaques métalliques soudées ensemble. Puis l'on a mesuré :

1) sa teneur en eau avec la sonde à neutrons,

2) son potentiel hydrique total avec des tensiomètres (Rose \& STERN, 1965).

\subsection{Evapotranspiration}

Ce paramètre est pratiquement le plus đélicat à estimer avec précision au niveau d'un écosystème. Aussi avons nous utilisé trois approches différentes pour tenter de i'évaluer avec un maximum de précision.

\subsection{Détermination de la transpiration par la méthode au tritium}

Cette méthode consiste à injecter une quantité connue de tritium dans un arbre et à suivre son élimination en fonction du temps au niveau des organes excréteurs (feuilles ou aiguilles). Elle permet de déterminer la transpiration réelle de l'arbre (KLine, 1970). 
Avant d'êrre utilisée sur le pin pignon, cette technique a fait l'objet d'une étude méthodologique (IBRAHIm et al., 1979) et les résultats d'une saison de végétation ont été analysés dans un travail antérieur (IвRAhim et al., 1980).

Au cours des sept mois de la saison de végétation de 1978, nous avons injecté du tritium dans 14 arbres, représentatifs du peuplement, à raison de deux arbres par période. Chaque expérience a duré de 3 à 5 semaines durant lesquelles l'on a échantillonné régulièrement les arbres, extrait l'eau des échantillons et mesuré l'activité spécifique.

a
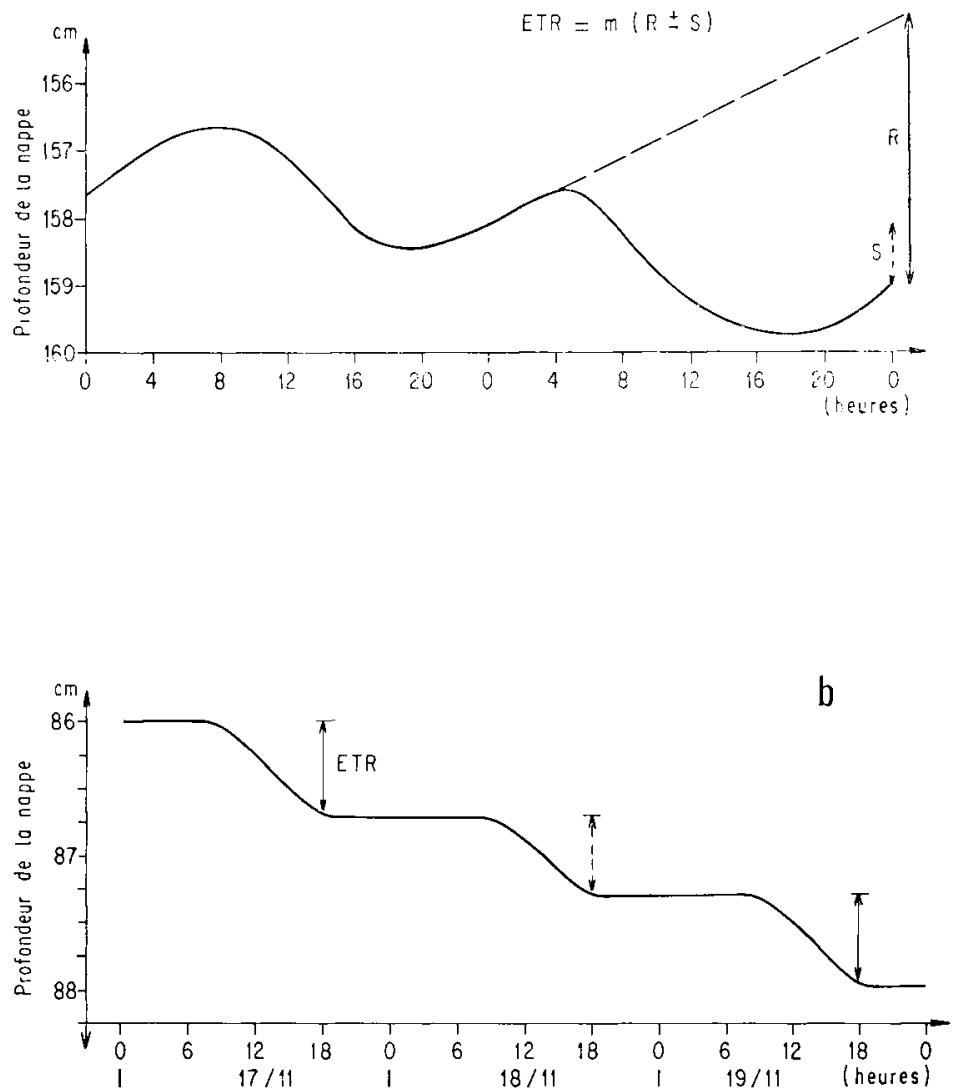

FIG. 1

Oscillations journalières de la nappe et calcul de l'ETR

Daily fluctuations in water table and ETR assessment

a - Recharge de la nappe à partir du canal d'irrigation, parallèlement à la ponction par transpiration.

Water table recharge from irrigation canal associated with uptake by transpiration.

b - Evolution de la nappe résultant uniquement de la transpiration, le canal d'irrigation et la nappe étant en équilibre.

Water table evaluation as a result of ETR while irrigation canal and water table are in equiubrium. 


\subsection{Analyses des fluctuations quotidiennes de la nappe}

La nappe phréatique oscille en permanence. Ce fait est bien connu et a été déjà utilisé par JAWORSKI (1969), URIE (1971) ou AUSSENAC (1972) pour chiffrer la transpiration de peuplements forestiers.

Du fait de l'interférence du canal, longeant le peuplement, l'évolution classique par paliers successifs qui caractérisent les états d'équilibre se trouve parfois modifiée pour faire place à un cycle sinusoïdal résultant d'une recharge simultanée à la transpiration.

En période de recharge à partir du canal, les limnigrammes prennent laaspect classique, de forme sinusoïdale, bien décrit par JAwORSKI (1969) et CALLEDE et al. (1978). La figure 1 montre une telle situation durant deux journées consécutives. Cette figure représente bien la recharge en dehors de toute perte par transpiration, durant la période nocturne $\{0$ à 4 ). Son extrapolation à la journée donne la hauteur de recharge $R$. Soit $S$ la différence de niveau de la surface piézométrique en 24 heures, résultat d'un gain ou d'une perte, $m$ la porosité de drainage, l'on peut alors exprimer l'ETR par la formule suivante :

$$
\mathbf{E T R}=\mathrm{m}(\mathrm{R} \pm \mathrm{S})
$$

Dans la deuxième situation, la plus classique, l'ETR se détermine directement par la différence de niveau quotidienne de la surface de la nappe, multipliée par la porosité de drainage.

\subsection{Estimation à partir du bilan hydrique}

Au niveau stationnel, l'équation du bilan hydrique peut s'écrire :

d'où :

$$
\{\Delta S\}_{0}^{z}=\operatorname{Pn}-\mathrm{ETR}-\theta \mathrm{z} \Delta \mathrm{t}
$$

$$
\mathrm{ETR}=\operatorname{Pn} \pm\{\Delta\}_{0}^{\pi}-\theta \Delta \mathrm{t}
$$

avec :

Pn : pluie nette arrivant au sol, donc

$\mathrm{Pn} \quad$ : $\mathrm{Pi}$ - Interception

$$
\text { : } \mathrm{Eg}+\mathrm{Ec}
$$

$\{\Delta S\}_{11}^{\mathbf{z}}:$ variation de stock d'eau sur épaisseur $z$ ae sol

$\theta \mathrm{z} \quad$ : flux moyen à travers la côte $\mathrm{z}$. Si l'on oriente $\mathrm{Oz}$ vers le bas, ce flux sera compté positivement dans ce sens, c'est-à-dire en cas de drainage.

Il suffit donc d'effectuer les mesures et les calculs suivants : la somme des pluies arrivant au sol $\mathrm{Pn}$ pendant la période considérée, la variation de stock d'eau moyen de la parcelle $(\Delta S)$ dans les premiers $60 \mathrm{~cm}$ du sol (zone de la quasi totalité des racines des arbres) et le flux $0 \mathrm{z}$ (drainage ou remontée capillaire) calculé entre 60 et $90 \mathrm{~cm}$ de profondeur en appliquant la loi de DARCY généralisée.

La figure 2 représente l'évolution de l'évapotranspiration du peuplement au cours des périodes de végétation de deux années consécutives. 

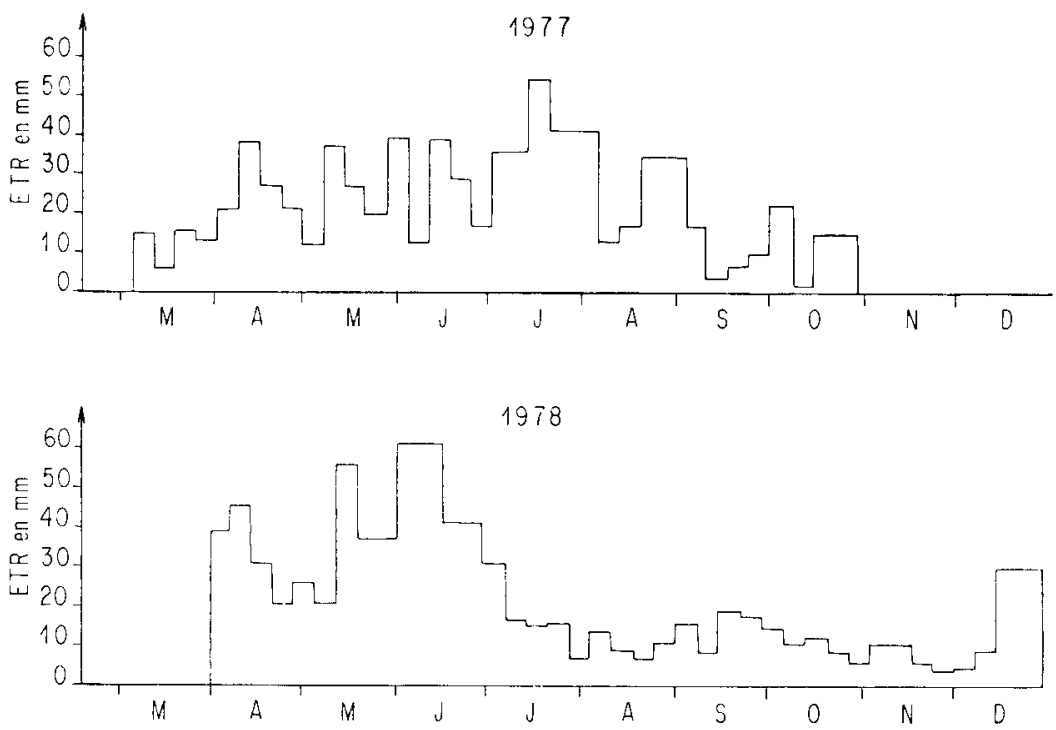

FiG. 2

Evolution saisonnière de l'évapotranspiration calculée à partir de la méthode du bilan hydrique

Seasonal evolution of ETR evaluated using the water balance method

Quantitativement elle représente respectivement 603 et $624 \mathrm{~mm}$ durant deux périodes végétatives successives, donc des valeurs sensiblement identiques malgré des pluviosités nettement différentes.

Ce fait confirme l'importance de la nappe dans le budget hydrique de la station en général et dans l'alimentation en eau des arbres en particulier. Ce rôle a déjà été signalé par Daudet \& Valancogne (1976) et par Reicosky et al. (1977).

Mais les valeurs de l'ETR déterminées par cette méthode présentent une certaine incertitude dans le cas présent par suite de l'existence permanente d'une zone saturée à partir de 50 à $60 \mathrm{~cm}$ de profondeur. Ceci peut fausser les relevés tensiométriques, dont les variations étaient par ailleurs minimes, erreur qui se répercute sur le résultat final, c'est-à-dire l'ETR (DAUdeT \& VAchaud, 1977).

\subsection{Comparaison des trois approches}

Nous avons résumé sur le tableau 1 l'ensemble des estimations de l'ETR pour les années 1977 et 1978 à partir des méthodes utilisées.

Les résultats obtenus par le bilan hydrique sont régulièrement inférieurs à ceux fournis par les deux autres méthodes. Cette diminution de 30 à 40 p. 100 par rapport aux deux autres résulte essentiellement de la tranche de sol prise en considération dans son établissement. En effet ce bilan ne porte que sur un profil de $63 \mathrm{~cm}$ 


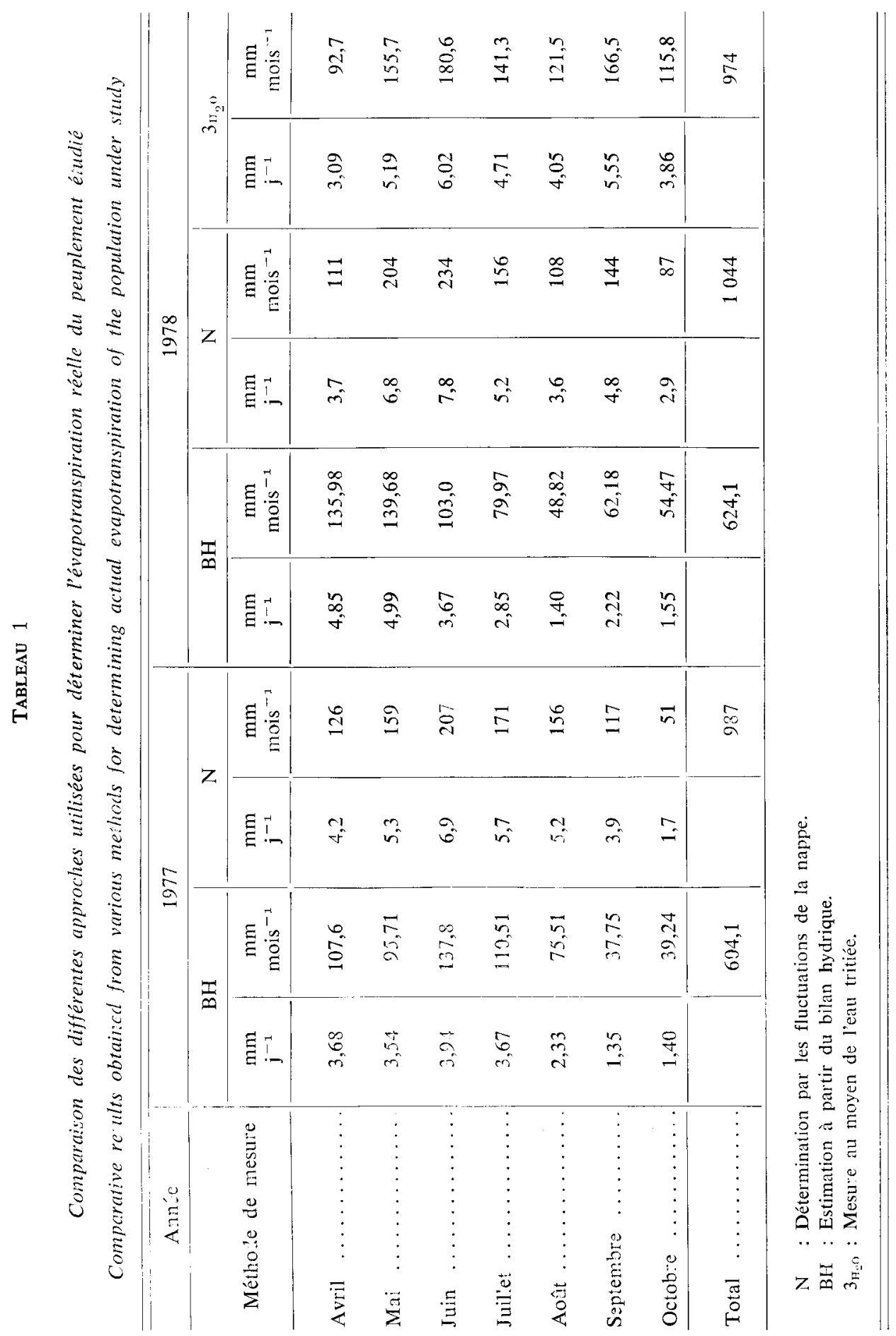


d'épaisseur et toutes les ponctions d'eau directes effectuées par le système racinaire à des profondeurs plus élevées ne sont pas prises en considération. Par suite de la proximité de la nappe, l'utilisation des tensiomètres n'étant possible que dans une tranche de sol ne dépassant pas 60 à $90 \mathrm{~cm}$. Un tensiomètre implanté à $120 \mathrm{~cm}$, n'a permis d'enregistrer aucune variation exploitable car il était soit dans la nappe, soit dans la frange capillaire. Daudet \& Vachaud (1977) ont bien analysé ce problème et indiqué les limites de la méthode du bilan hydrique dans le cas des transferts rapides sans variations importantes de la teneur en eau en zone saturée.

Pour toutes ces raisons, cette première approche n'est pas valable dans les conditions particulières du système étudié. Mais elle permettra de différencier au niveau du bilan les différentes sources de l'eau évapotranspirée.

Les deux autres approches, elles, donnent des résultats sensiblement voisins : $1044 \mathrm{~mm}$ par la méthode basée sur les fluctuations de la nappe et $974 \mathrm{~mm}$ par la méthode au tritium. Au cours de 3 années successives l'ETR établie à partir de la nappe est relativement constante, avec les valeurs respectives de 1062,987 et $1044 \mathrm{~mm}$ de 1976 à 1978.

Néanmoins cette dernière approche présente une certaine inexactitude introduite par l'interférence de la roubine sur la nappe. L'instabilité résultant des impératifs agronomiques du canal a entraîné des variations continuelles du niveau de la nappe qui ont sensiblement réduit les périodes d'estimation de l'ETR.

Le résultat final représente donc une extrapolation à partir d'un nombre limité d'observations précises. Il est donc possible, que certaines de ces observations ne correspondent pas, dans la réalité, à des périodes caractéristiques. Mais l'excellente reproductibilité annuelle et la bonne concordance constatée en 1978 avec la méthode au tritium incitent à faire confiance aux estimations obtenues à partir de cette méthode.

\section{5. - Résultats et discussion}

Tous ces paramètres ont été étudiés et quantifiés au cours de la période végétative des années 1977 et 1978 (avril à octobre).

En plus de tous les paramètres de la formule (1) nous analyserons les trois variables suivantes :

- précipitations sous couvert (Pn),

- transpiration (T),

- évaporation physique (E),

et les valeurs de l'évapotranspiration réelle (ETR) déterminées à partir de la nappe $\left(E_{N}\right)$ et du bilan hydrique $\left(\operatorname{ETR}_{1 B H}\right)$.

Nous avons regroupé sur le tableau 2 les valeurs mensuelles et totales des différents paramètres de l'économie de l'eau pour les périodes de végétation de ces deux années. 


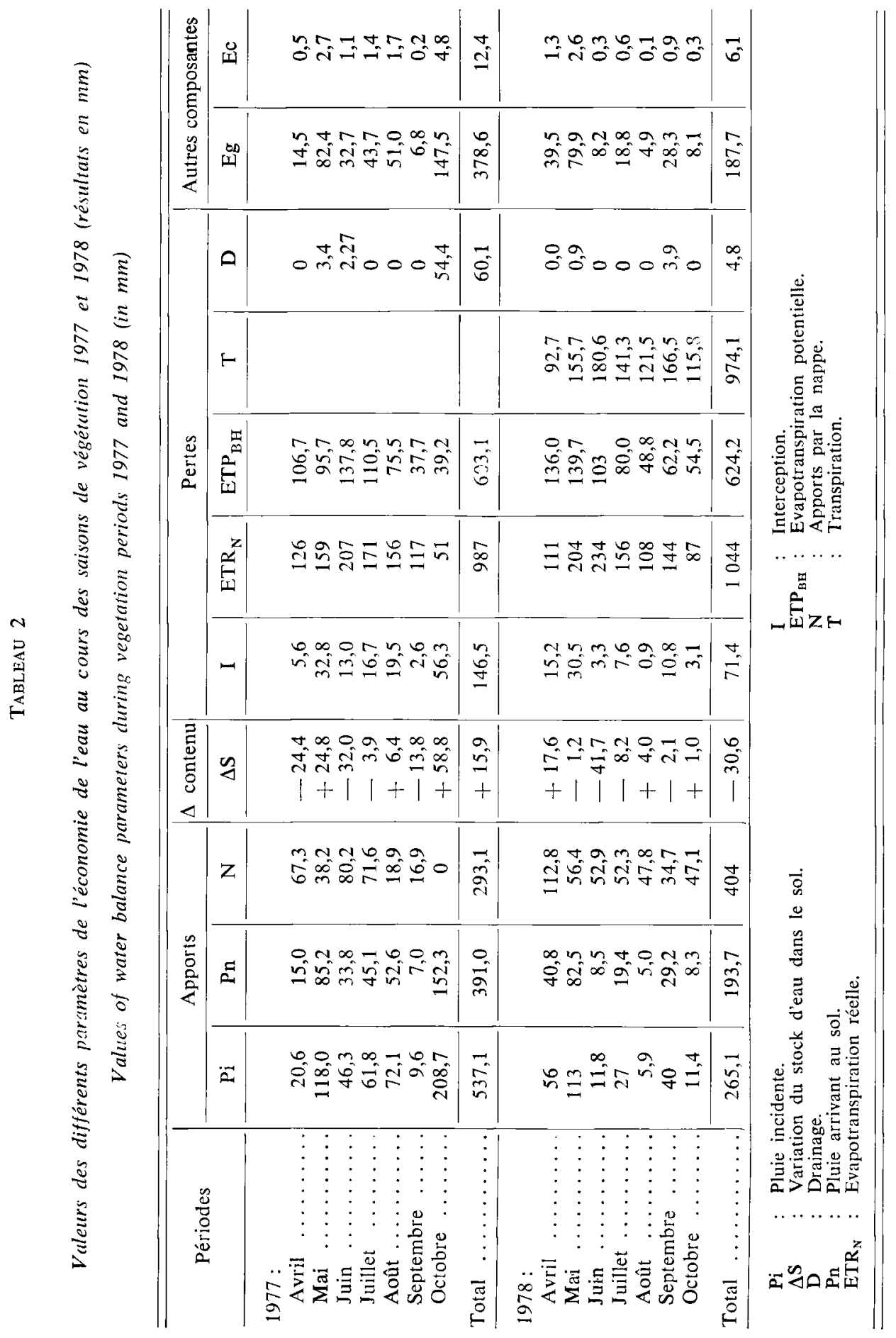




\subsection{Le bilan de l'année 1977}

Pendant la période végétative de 1977, le système plante-sol a bénéficié de $830 \mathrm{~mm}$ $(537+293)$ d'eau par l'intermédiaire des précipitations et de la remontée capillaire dans la zone colonisée par la quasi totalité des racines. Cette dernière $(N=293 \mathrm{~mm})$ représente 35 p. 100 des apports totaux, alors que les précipitations $(P=537 \mathrm{~mm})$ représentent 65 p. 100.

Une fraction de l'apport atmosphérique, équivalente à $146 \mathrm{~mm}$ (27 p. 100 des précipitations) est interceptée (I) par la couverture végétale. Le restant $\left(P_{n}\right)$ arrive au sol par écoulement ou égouttement. Il représente $391 \mathrm{~mm}$ ou 73 p. 100 des pluies. Une lame d'eau de $60 \mathrm{~mm}$ environ, ou 11 p. 100 de la pluie incidente sera drainée hors de la zone des racines (D). Elle va alimenter la nappe et pourra éventuellement être absorbée au niveau du chevelu racinaire qui coiffe le pivot terminal.

En effet, l'évapotranspiration totale établie directement à partir des fluctuations de la nappe $\left(\right.$ ETR $\left._{\mathrm{N}}\right)$ s'élève à $987 \mathrm{~mm}$. Or $603 \mathrm{~mm}$ seulement $\left(\right.$ ETR $\left._{\mathrm{BH}}\right)$, soit $73 \mathrm{p} .100$ de l'apport total $(830 \mathrm{~mm})$ ou 111 p. 100 des précipitations $(537 \mathrm{~mm})$ proviennent des 60 premiers centimètres du sol.

Les $384 \mathrm{~mm}$ restants proviennent donc d'une absorption par la partie du pivot en contact direct avec la nappe, car peu de racines secondaires susceptibles d'absorber de l'eau couvrent le pivot entre $60 \mathrm{~cm}$ et les ramifications terminales (CABANETTES, 1979). C'est d'ailleurs pour cette raison que nous avons été obligés de déterminer l'ETR par d'autres méthodes que le bilan hydrique. Ces $384 \mathrm{~mm}$ fournis par la nappe représentent 39 p. 100 de l'ETR, les autres 61 p. 100 provenant des premiers $60 \mathrm{~cm}$ du sol.

Durant cette période, la variation du stock d'eau du sol $(\Delta S)$ a été de $15 \mathrm{~mm}$, c'est-à-dire que la réserve en eau du sol a augmenté de $15 \mathrm{~mm}$ entre le début et la fin de la période de végétation.

\subsection{Bilan de l'année 1978 (figure 3)}

En procédant de la même manière pour 1978, l'on obtient les valeurs suivantes pour le bilan de l'eau :

\section{Apports}

Pluies incidentes

Pluies sous couvert

Ecoulement

Egouttement

Remontée capillaire

Total des apports

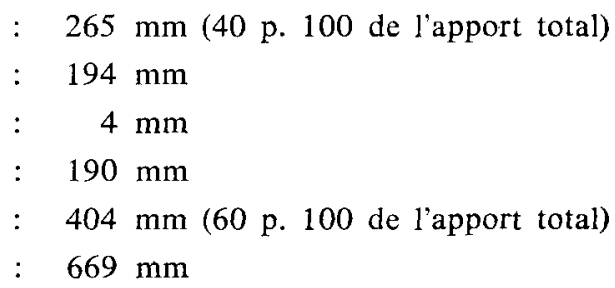


Pertes

Interception

: $\quad 71 \mathrm{~mm} \mathrm{(27} \mathrm{p.} 100$ de la pluie incidente)

Drainage

$5 \mathrm{~mm}(1 \mathrm{p} .100$ de l'apport total ou 2 p. 100 de la pluie incidente)

ETR (total à partir de la nappe) ETR $_{X}: 1044 \mathrm{~mm}$

ETR (partie fournie par le sol) ETR BII $_{\text {: }} 624 \mathrm{~mm}(60$ p. 100 de l'ETR totale)

Transpiration des arbres (T) : $974 \mathrm{~mm}(93$ p. 100 de l'ETR totale)

Fluctuation du stock d'eau du sol $(\Delta S):-30 \mathrm{~mm}$ (3 p. 100 de l'ETR totale)

Avec la détermination directe de la transpiration des arbres par la méthode au tritium, nous pouvons pour cette année faire un pas de plus dans la quantification des différentes flux de l'eau dans l'écosystème. En effet, connaissant l'ETR totale, $1044 \mathrm{~mm}$ et la transpiration par les pins $974 \mathrm{~mm}$, nous pouvons attribuer la différence, soit $70 \mathrm{~mm}$, à l'évaporation physique à partir du sol. Comme pour l'année 1977, l'on peut aussi à partir des deux estimations de l'ETR, calculer la part revenant à la nappe qui équivaut à $1044-624=420 \mathrm{~mm}$ (ou 40 p. 100 de l'ETR).

\subsection{Comparaison des deux années}

Les fluctuations interannuelles du bilan hydrique sont essentiellement dues à la variabilité des précipitations et des autres conditions climatiques locales.

Les premières, par les quantités de pluic et lcur répartition le long de l'année, sont les variables essentielles des apports d'eau; par contre, les potentialités d'apport par la nappe peuvent être considérés comme constantes et optimales. Au niveau des pertes, ce sont les facteurs climatiques responsables de l'évapotranspiration (rayonnement, température, humidité relative et vent) qui interfèrent essentiellement sur le bilan de l'eau.

La différence entre les deux années est caractérisée par une forte ponction dans la nappe au cours de l'année 1978. Ce prélèvement se retrouve à la fois dans la remontée capillaire (404 mm en 1978 contre $293 \mathrm{~mm}$ en 1977) et à un degré moindre dans la ponction directe $(420$ contre $384 \mathrm{~mm})$. Elle résulte essentiellement d'une grande sécheresse durant la période de végétation de lannée 1978 (275 mm de pluie), par rapport à l'année 1977 (537 mm de pluie) alors que les précipitations totales de ces deux années sont assez proches : 759 et $691 \mathrm{~mm}$ respectivement. Ce déficit en pluie se traduit par un faible drainage en $1978(5 \mathrm{~mm})$ par rapport à $1977(60 \mathrm{~mm})$. Mais à part ces différences à l'intérieur des divers compartiments, le bilan global reste reiativement voisin et stable. Ceci confirme entièrement le rôle de réservoir joué par la nappe.

\subsection{Discussion générale et Conclusions}

Les bilans établis pour les deux années 1977 et 1978 présentent un certain nombre de limitations. Les unes sont inhérentes aux méthodes expérimentales d'estima- 


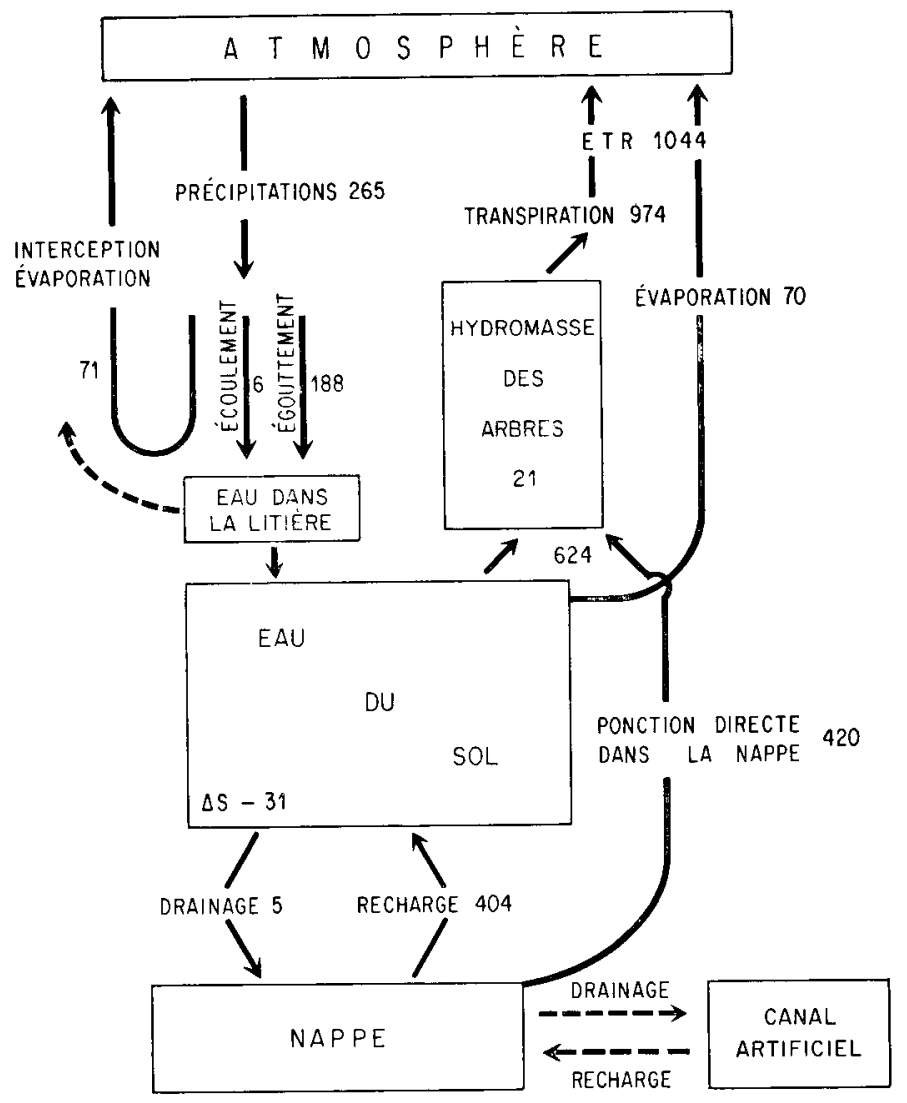

FIG. 3

Cycle et budget de l'eau de l'écosystème Pinus pinea $L$.

étudié au cours de la saison de végétation (avril à octobre, 7 mois) de l'année 1978

Water cycle and budget of Pinus pinea $L$. ecosystem investigated during vegetation period April-October 1978 (7 months)

Les compartiments sont encadrés, les flux représentés par des flèches pleines si leur valeur est déterminée, brisées s'ils n’ont pas été chiffrés.

$\Delta \mathrm{S}=$ Variation totale du stock d'eau du compartiment sol au cours de la période de végétation.

Les résultats sont exprimés en mm d'eau.

Compariments are framed. Flows are represented by full arrows when their value is determined, and by discontinuous arrows when undetermined.

$\Delta S=$ Total variation in soil compartment water reserve during vegetation period. Results expressed in mm water. 
tion des différents paramètres utilisés, les autres résultent de la faible durée de l'observation de certains paramètres empêchant toute vérification statistique des résultats.

Ainsi, dès l'estimation de la pluviométrie et de l'interception, une incertitude peut résulter à la fois des caractéristiques des pluviographes et pluviomètres et de leur implantation à une certaine distance de la forêt ou sous les arbres.

Si la précision des humidimètres à neutrons est excellente et si nous n'avons après une investigation prolongée retenu qu'un nombre restreint de profils les plus représentatifs de la station, il subsiste une certaine imprécision due au large éventail d'humidité rencontré au cours des années de mesure. En effet, il est bien connu que la sphère dans laquelle s'effectue la mesure neutronique est directement fonction de l'humidité du milieu et a donc varié fréquemment durant cette étude.

Pour le calcul de l'ETR à partir des observations de la nappe, nous n'avons pas pu utiliser l'intégralité des observations, mais procédé par extrapolation au mois, d'un certain nombre d'observations hebdomadaires.

La précision de l'estimation de la transpiration par le tritium peut être considérée comme bonne au niveau de l'arbre (Iвrahim et al., 1979). Elle présente par contre une certaine approximation lors de lextrapolation au niveau de la station (IBRAHIM, 1979).

Par contre la conductivité hydrique qui est un facteur primordial pour le calcul du flux en profondeur (drainage ou remontée capillaire) a été mesurée sous forêt dans une placette isolée. Il n'était pas possible pour des raisons pratiques évidentes de la répéter pour chaque profil neutronique. De même la porosité de drainage ou coefficient d'emmagasinement, a été calculée à partir d'un certain nombre d'études ponctuelles des limnigrammes. La moyenne de 15 p. 100 obtenue présente des écarts assez importants pouvant atteindre 35 p. 100.

Enfin nous avons, au niveau du budget, fait l'impasse sur l'interception par la litière qui se situe aux alentours de 4 p. 100 des précipitations et négligé de faire une étude détaillée de l'évaporation physique.

Malgré toutes ces limites et en utilisant des approches différentes, nous avons abouti à un bilan annuel équilibré. Les entrées et les sorties sont voisines, les approches multiples pour déterminer un même flux donnent des résultats concordants, comme c'est le cas pour l'estimation de l'ETR et de la transpiration des arbres ou de l'ETP et de l'ETR. Les différences que l'on rencontre en utilisant différentes méthodes sont faibles, avec des écarts de l'une à l'autre de l'ordre de 10 p. 100 au cours des deux années consécutives d'observation.

L'on note ainsi l'excellente concordance entre l'évapotranspiration calculée à partir des fluctuations de la nappe, la transpiration des pins et l'évapotranspiration potentielle (ETP) calculée à l'aide de formules bioclimatiques classiques pour trois années successives (méthode du Piche corrigée de Boucher, 1961) et corrigée pour les écosystèmes forestiers d'après RUTTER (1968) : 
Pluviométrie

ETP calculée

ETP corrigée

ETR

Transpiration
1976

1977

1978

\author{
$543 \mathrm{~mm}$ \\ $755 \mathrm{~mm}$ \\ $944 \mathrm{~mm}$ \\ $1062 \mathrm{~mm}$
}

$537 \mathrm{~mm}$

$712 \mathrm{~mm}$

$890 \mathrm{~mm}$

$987 \mathrm{~mm}$
$401 \mathrm{~mm}$

$909 \mathrm{~mm}$

$1136 \mathrm{~mm}$

$1044 \mathrm{~mm}$

$974 \mathrm{~mm}$

La relative similitude des résultats permet de conclure que dans les conditions étudiées, le peuplement bénéficie toujours d'une bonne alimentation en eau grâce aux précipitations et surtout à la nappe phréatique constamment accessible. Au cours des années à précipitations importantes, l'on assiste pour ainsi dire à une surconsommation d'eau, l'ETR étant supérieure à l'ETP. Par contre, en 1978, année plus sèche, l'ETR était inférieure à l'ETP indiquant un certain frein à l'alimentation hydrique du peuplement.

Reste le fait que le budget a été établi pour la période végétative, donc sept mois. Pour essayer de l'estimer pour lannée entière, nous avons établi pour les années 1976, 1977, 1978, le rapport :

ETP calculée pendant 7 mois

ETP année entière

Ce rapport est respectivement de 69,74 et 80 p. 100 pour les trois années d'observation, avec une moyenne de 75 p. 100. Comme l'ETP et l'ETR sont voisines dans les conditions de la station, l'on peut évaluer l'ETR moyenne des années 1977 et 1978 à $1319 \mathrm{~mm}$. Durant cette période, l'apport par les pluies est de $725 \mathrm{~mm}$, avec 529 arrivant au sol. Quant à la remontée capillaire elle reste de $348 \mathrm{~mm}$, le profil étant saturé les autres mois.

L'on obtient alors :

Apports

$$
\begin{array}{ll}
\text { Pluies (Pi - I) } & 529 \mathrm{~mm} \\
\text { Remontées capillaires } & 348 \mathrm{~mm} \\
\cline { 2 - 2 } & 877 \mathrm{~mm}
\end{array}
$$

Pertes

$$
\begin{array}{lr}
\text { ETR } & 1319 \mathrm{~mm} \\
\text { Ponction dans la nappe } & 442 \mathrm{~mm}
\end{array}
$$

Les valeurs annuelles sont donc légèrement plus élevées, ce qui est logique, mais parallèles à celles obtenues pour les sept mois de la période de végétation.

Cette dernière est donc bien représentative de l'ensemble du cycle et du budget de l'eau de la station étudiée. 
En ce qui concerne la transpiration, déterminée en 1978, et s'élevant à $974 \mathrm{~mm}$ pendant la saison de végétation, elle représente en moyenne par arbre, $12,17 \mathrm{~m}^{3}$ soit entre 40 et 75 litres par jour, selon l'époque de l'année.

Calculé par hectare, cela représente $9740 \mathrm{~m}^{3}$. Ces chiffres sont très élevés si on les compare à ceux de KüNTZle \& Mitscherlich (1977) obtenus en Forêt Noire sur d'autres arbres, par d'autres méthodes, mais les différences s'expliquent par le fait que dans le cas présent le Pin pignon bénéficie d'un apport direct par la nappe d'environ 40 p. 100 de l'eau transpirée.

Si l'on établit le coefficient de transpiration (ou efficience transpiratoire) de la productivité : eau transpirée ha-1, en litres ( 9740000 litres), par $\mathrm{kg}$ de matière sèche produite $(15390 \mathrm{~kg}$ ), en se basant sur les données de CaBanetTes (1979), on obtient un chiffre de 633 .

Comparée aux données rassemblées par LARCHER (1980), cette valeur est également très élevée. Ici aussi, on l'explique par une consommation de luxe d'eau facilement disponible.

Reçu pour publication le 20 octobre 1981.

\author{
Summary \\ Water balance in a Pinus pinea $L$. ecosystem \\ of the Mediterranean littoral
}

In the frame of an integrated research project over the ecology of Pinus pinea, the water budget of a 33 years old stand has been studied.

The following fluxes and compartments of the water cycle have been quantified : input to the stand by rain ; canopy interception, soil water fluctuations; output from the soil to the water table by drainage and input from the water table by capillary rising; seasonnal variation of the ground water table and finally, output to the atmosphere by the transpiration of the trees, determined by using tritiated water. seasons.

The complete water budget of the stand was established for two consecutive growing

The measured evapotranspiration amounts nearly to the same value as the potential evapotranspiration, calculated by bioclimatical equations. This agrees with the good growing and colonisation potentialities of Pinus pinea, along the mediterranean coast, if it always benefits of good water supply provided by easy available ground water.

Key-words

Ecosystem, water budget, Pinus pinea

\title{
Références bibliographiques
}

Aussenac G., 1972. Etude de l'évapotranspiration réelle de quatre peuplements forestiers dans l'Est de la France. Ann. Sci. for., 29 (3), 369-389.

Benecke P., Mayer R., 1971. Aspects of soil water behavior as related to beech and spruce stands. Some results of the balance investigation. Integrated experimental ecology, Ellenberg H. (ed.), Springer-Verlag, 153-163. 
Bouchet R.J., 1961. Signification et portée agronomiques de l'évapotranspiration potentielle. Ann. agron., 112 (1), 51-63.

Blum H.P., Munnich K.O.U., Zimmermann U., 1966. Das Verhalten des Wassers in ciner Lössparabraunerde unter Laubwald. Z. Pflanzenernähr. Düng-Bodenkd., 112, 156-168.

Cabanettes A., 1979. Croissance, biomasse et productivité de Pinus pinea L. en Petite Camargue. Thèse de $3^{*}$ cycle, U.S.T.L. Montpellier (France), $175 \mathrm{p}$.

Callede J., Hallaire M., Daudet F.A., 1978. Oscillations journalières de la profondeur des nappes en l'absence de précipitations. Ann. agron., 29 (2), 111-122.

Couchat P., 1974. Mesure neutronique de l'humidité des sols. Thèse de doctorat ès-sciences, Toulouse, Université Paul-Sabatier, 123 p. + annexes.

Daudet F.A., Valancogne Ch., 1976. Mesure des flux profonds de drainage ou de remontée capillaires. Leur importance dans le bilan hydrique. Ann. agron., 27 (2), 165-182.

Daudet F.A., Vachaud G., 1977. La mesure neutronique du stock d'eau du sol et de ses variations. Application à la détermination du bilan hydrique. Ann. agron., 28 (5), 503-519.

Ettehad R., Lossaint P., Rapp M., 1973. Recherches sur la dynamique et le bilan de l'eau des sols des deux écosystèmes méditeranéens à chêne vert. In : Nouveaux documents pour une étude intégrée en écologie du sol. C.N.R.S. ed., R.C.P. 40, Paris, 194-279.

Fardjah M., Lemee G., 1980. Dynamique comparée de l'eau sous hêtraie et dans des coupes nues ou à Calamagrostis epigeios en forêt de Fontainebleau. Bull. Ecol., 11 (1), 11-31.

Halldin S., Jansson P.E., 1976. Water Model - Swedish coniferous Forest Project, P.M., 1976-02-24, Uppsala, 55 p. (ronéo).

Huttel C., 1972. Estimation du bilan hydrique dans une forêt sempervirente de basse Côte-d'Ivoire. Isotope and radiation in soil plant relationships including forestry. I.A.E.A. Vienna, 439-452.

IbRaHim M., 1979. Recherche sur la dynamique et le bilan de l'eau d'un écosystème à pin pignon (Pinus pinea L.) sur sable dunaire. Thèse U.S.T.L., Montpellier (France), 271 p.

Ibrahim M., RapP M., 1979. Variation spatio-temporelle de la salinité du sol d'un peuplement de pins pignons (Pinus pinea L.) du littoral méditerranéen. Oecol. Mediterr., 4, 49-60.

Ibrahim M., Berger A., Rapp M., 1979. Détermination de la transpiration au moyen de l'eau tritiée. Méthodologic et validité des résultats. Application à Pinus pinea L. Plant. and Soil, 52 (2), 291-301.

Ibrahim M., Rapp M., Berger A., Lossaint P., 1980. Evaluation de la transpiration d'un peuplement de Pinus pinea L. en condition naturelle. Acta oecol., Oecol. Plant., 1 (15), n" 4, 395-407.

JAworski J., 1969. Evapotranspiration of plants and fluctuations of the ground water table. A.I.S.H., Symposium de Wageningen, Vol. 2, 730-739.

Kline J.R., Martin J.R., Jordan C.F., Koranda J.J., 1970. Measurement of transpiration in tropical trees using tritiated water. Ecology, 5 (6), 1068-1073.

KüNstle E., Mitscherlich G., 1977. Photosynthese, Transpiration und Atmung in ei ${ }^{1} \mathrm{~cm}$ Mischbestand im Schwarzwald. IV Teil : Bilanz Allg. Forst. Jagdztg, 148, 227-239.

Larcher W., 1980. Oekologie der Pflanzen, U.T.B. Ulmer, Stuttgart, 400 p.

Lossaint P., Rapp M., 1978. La forêt méditerranéenne des chênes verts. In : M. Lamotte, F. Bourliere (ed.), Problèmes d'Ecologie, Ecosystèmes Terrestres, Masson, Paris, 129-185.

Molchnnov A.A., 1971. Cycle of atmospheric precipitation in different types of forest of naturel zones of the U.S.S.R. In : Productivité des écosystèmes forestiers, U.N.E.S.C.O.-Paris, 49-68.

Rapp M., Iвrahim M., 1978. Egouttement, écoulement et interception des précipitations par un peuplement de Pinus pinea L. Oecol. Plant., 13 (4), 321-330.

Rapp M., Leclerc M.-Cl. Lnscaint P., 1979. The nitrogen economy in a Pinus pinea L. stand. For. Ecol. and Management, 2 (3), 221-231. 
Reicosky D.C., Doty C.W., Campbell R.B., 1977. Evapotranspiration and soil water movement beneath the root zone of irrigated and non irrigated millet (Panicum miliaceum). Soil Sci., 124 (2), 95-101.

Rose C.W., Stern W.R., 1965. The drainage component of the water balance equation. Aust. J. Soil Res., 3, 95-100.

RutTer A.J., 1968. Water consumption by forests. In : Water deficits and plant growth, Vol. II, Plant water consumption and response (T.T. KozlowkI, ed.), Academic Press, New York, 23-84.

ScHNock G., 1970. Le bilan d'eau et ses principales composantes dans une chênaie mélangée calcicole de Haute Belgique (Bois de Virelles-Blaimont). Thèse Univ. Libre de Bruxelles, 352 p.

SchNock G., 1972. Contenu en eau d'une phytocénose et bilan hydrique de l'écosystème : chênaie de Virelles. Oecol. Plant., 7 (3), 205-226.

URIE D.H., 1971. Estimated ground water yield following strip cutting in pine plantations. Water Resour. Res., 7 (6), 1497-1509. 